INTERNATIONAL JOURNAL OF RESEARCHES IN BIOSCIENCES, AGRICULTURE AND TECHNOLOGY

(C) VISHWASHANTI MULTIPURPOSE SOCIETY (Global Peace Multipurpose Society) R. No. MH-659/13(N)

\title{
OPLC (OPTIMUM PERFORMANCE LAMINAR CHROMATOGRAPHY) A BRIDGE BETWEEN HPLC AND HPTLC
}

\author{
G. Barabde \\ Institute of Science, Fort, Mumbai \\ grbarabde@gmail.com
}

\begin{abstract}
:
Chromatographic, Electro chromatographic and different spectroscopic technique play a crucial role in isolating and identifying the potential ingredients in medicinal plants. For chromatographic fingerprinting TLC/HPTLC and HPLC are used for analysis. The optimal separation distance of TLC/HPTLC plates and the use of only one layer volume limit the number of components that can be separated. While in HPLC the sample preparation of medicinal plants before injection of sample is tedious process and also there are chances of losing any component. To overcome limitations of both techniques in case of medicinal plants OPLC is a powerful separation technique that uses a solvent pump into a pressurized ultra micro chamber containing an analytical or preparative planer adsorbent bed to separate components' of a mixture. OPLC require no or little sample preparation as in TLC and can use the full length of layer for analytical and preparative separation as in HPLC.
\end{abstract}

Keywords: OPLC, Laminar, Pressurized, ultra micro

\section{Introduction:}

Column Chromatography is widely used in both analysis and preparative isolation of natural and synthetic organic samples. High pressure column liquid chromatography uses one dimensional (1D) format. Optimum performance laminar chromatography (OPLC), in contrast, is a pumped flow chromatography system that uses a planar $2 \mathrm{D}$ column format. This can increase throughput by allowing sideby-side analysis of several samples in a single run, provide added loading capacity by using the whole column width for a single sample and improve separation efficiency by allowing twodimensional (2D) chromatography directly on a square Column. The name OPLC is described as over pressured layer chromatography or forced flow TLC.

This technique was developed in late 1970s. The instrument at that time used nuts and bolts to compress a modified TLC plate between metal or glass sheets and there was no control over the pressure applied. Later the TLC plate was modified by adding a sealant to the edges of the plate. To distribute the solvent homogeneously across the layer, it was also necessary to scrape a horizontal line of silica from the plate directly below the solvent inlet. All these factors and many more could not popularize this technique. The modern OPLC technique has overcome these factors and made the technique advanced and user friendly. The factor which has made OPLC a wonderful technique is the introduction of electronic controls which monitors the chromatographic conditions and effective separation in minimum $43 \mathrm{sec}$. The readymade flat columns have a high precision positioning and a more regular seal.
The flowing eluent wall technology (FEW) dramatically reduces disturbances at the layer edge and the inlet Site This review article will describe the basics of modern OPLC instrumentation and the separation techniques,

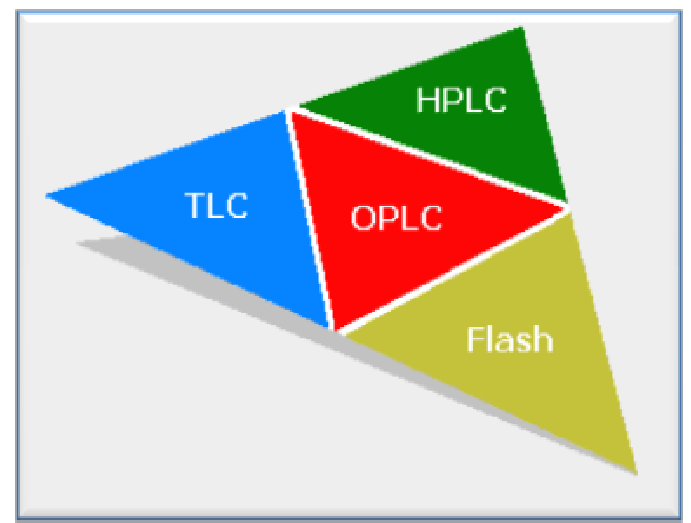

Figure. 1-

\section{INSTRUMENTATION:}

The basis of OPLC is similar to that of other chromatographic techniques in that a pump is used to force a liquid mobile phase through a stationary phase, such as silica or a bonded-phase media (e.g., C8, C18, amino or nitrile). The uniqueness of OPLC lies in its column housing structure that allows flat planar columns to be used in the same way as cylindrical glass or stainless steel ones. An OPLC development chamber is consist of 1) A planer column i.e. a cassette constructed of a machined PTFE sheet. 2) An Aluminum support. 3) An electronically controlled hydraulic press. The planar column itself is a uniform sorbent bed layered on a glass or aluminum backing with an elastomeric polymer seal located at the periphery. The column is placed silica face-up in 
the cassette, in direct contact with the PTFE sheet. This, in turn, is placed inside the hydraulic press of the development chamber. Upon pressure, the PTFE sheet is pressed onto the sorbent bed and compresses the seal creating a solvent-tight system ready for chromatographic separations. Mobile phase is then pumped through connections on the development chamber to the sorbent bed. The machined PTFE sheet is responsible for distributing solvent to particular zones on the column and recovering it from the sorbent bed as a chromatographic separation is performed.

A cassette comprises an aluminum base plate and a PTFE cover.The cassette has two main functions: creating solvent-tightness under compression and determining solvent flow within the sorbent bed. The PTFE sheet has 1 $\mathrm{mm}$ holes that allow flow to the sorbent bed. Micro channels (250 $\mu \mathrm{m}$ wide, $350 \mu \mathrm{m}$ deep) on the underside of the PTFE sheet direct and recover solvent from predetermined zones on the stationary phase. Different cassette designs provide different separation modes. With two standard cassettes it is possible to perform basic 1D separations, as well as bidirectional,2D and simultaneous four sample 2D development. Recently a new technology has been introduced known as flowing eluent wall (FEW). In this case a sample free eluent is pumped to the lateral walls of the column near the elastomer seal. This confines the sample to a central portion of the column and prevents sample stream from reaching wall. Hence band peak is improved.

\section{OPERATION OF INSTRUMENT:}

A basic OPLC unit can be configured into an HPLC system and can either be used online, in the same way as a standard cylindrical HPLC column, or off line, with a HPTLC.

In case of On line mode the OPLC unit is installed in an HPLC system like an HPLC column. . The programmable workstation of the HPLC system ensures column equilibration, sample injection, detection and monitoring of the separation.

In case of off line mode the sample applied directly on column without much sample preparation and quantity of sample is not limited by an injection loop as in HPLC. Sample components are not eluted from the sorbent bed, as the results of the separation and can be observed directly on the column as in case of HPTLC. With off-line OPLC, there is less 10 to 100 times less solvent consumption as sample preparation is reduced to a bare minimum and column equilibration/regeneration is not necessary. In some instances, applying crude sample mixtures to the column can lead to improved reproducibility as the number of sample pretreatment steps is limited and all of the sample components actually reach the stationary phase where the separation is performed.

The upper pressure limit of the pump is automatically set by the instrument so that the pump stops when the development is complete. After development is complete samples can either be maintained on stationary phase and analyzed by HPTLC detectors or they can be eluted out to an appropriate detector,

\section{ADVANTAGES OVER HPLC AND HPTLC:}

$>$ OPLC integrates the benefits of TLC/HPTLC and HPLC. It requires no or little sample preparation as in TLC and can use the full length of layer for analytical and preparative separation as in HPLC

$>$ OPLC plates are efficient, low cost and reusable for multiple runs.

- Easy coupling to various detectors for on-line detection.

$>$ Can couple with any analytical solvent delivery unit for on-line or off-line preparative and analytical separations.

\section{APPLICATIONS:}

OPLC technology has been used several disciplines including Plant research, Pharmaceutical development, drug metabolism, Forensic analysis such as drug abuse etc. A few examples are listed below.

\section{Pharmaceutical analysis:}

OPLC analysis of steroid intermediates and other active pharmaceutical ingredients has been adopted by different scientists for inprocess control, final purity, cleaning validation and stability testing (allylestrenol, ${ }^{1}$ levonorgestrel,2nandrolone, ${ }^{3}$ digoxin, ${ }^{4}$ phthaloylamlodipine, 5 norethisterone and ethinylestradiol).They have proved that OPLC method is better then HPLC and HPTLC.

Forensic science: The Finnish group of Pelander has developed an OPLC technique for high-confidence identification of drug abuse substances in urine samples. 6

Bioactive molecule research: Bacterial and fungal toxins and their metabolic by-products in food products are known to cause serious health problems in humans and animals and, therefore, require regulatory monitoring. Research have been done to develop methods for the detection and assay of mycotoxin metabolites in food stuffs, ${ }^{7}$ such as rice, ${ }^{8}$ wheat ${ }^{9}$, fish 10 and corn.11 by OPLC. 


\section{Conclusion:}

OPLC is a unique and innovative technique of chromatography for better separation with high resolution for complex samples.

\section{Acknowledgement:}

Nathan Bryson and Domitille Papillard, Bionisis SA, Le Plessis Robinson, France

\section{Refrences:}

1)A. Wiszkidenszky et al., J. Planar Chromatogr., 11, 463, (1998)

2)K. Ferenczi-Fodor et al., Pharmeuropa, 9, 736, (1997).

3)B. Bagocsi et al., J. Planar Chromatogr., 15, 252, (2002).

4) A. Nagy-Turak et al., A Proc. Int. Symp. on Planar Chromatogr., Planar Chromatgr. 2000, Sz Nyiredy and A. Kakuk, Eds., (Research Inst. Med. Plants, Budapest, Hungary, 2000), p. 26.
5) Z. Sziksay, Z. Végh and K. Ferenczi-Fodor, J. Planar Chromatogr., 11, 428, (1998).

6) (a) I. Ojanperä, K. Goebel and E. Vuori, J. Liq. Chrom \& Rel. Technol., 22, 161 (1999); (b) A. Pelander et al., J. Liq. Chrom \& Rel. Technol., 24, 1425, (2001); (c) A. Pelander et al., J. Anal. Tox., 27, 226, (2003).

7) 29. E. Papp et al., Microchemical J., 73, 39 (2002)

8) G. Katay, A. Szecsi and E. Tyiak, J. Planar Chromatogr., 14, 53, (2001).

9) E. Papp et al., J. Planar Chromatogr., 13, 328, (2000).

10) E. Papp et al., J. Planar Chromatogr., 12, 383, (1999).

11)K. H-Otta et al., J. Planar Chromatogr., 11, 370, (1998). 